\title{
Ion Sensitive Field Effect Transistor Performance Enhancement with High Bandgap Semiconductors
}

\author{
Sakineh Heidari, Mohammad Azim Karami, \\ School of Electrical Engineering, Iran University of Science and Technology, Tehran, Iran \\ sakineh_heidari@elec.iust.ac.ir
}

\begin{abstract}
:
This paper introduces high bandgap semiconductors to increase the sensitivity of Ion Sensitive Field Effect Transistor (ISFET) to concentration of hydrogen $(\mathrm{pH})$ of electrolyte. Silicon dioxide is used as the sensing film in the proposed device. The ISFET principle of performance based on electrolyteinsulator-semiconductor structure is explained. The simulation results show ISFET with GaAs substrate has in average 2.3 times more sensitivity to $\mathrm{pH}$ changes in compare with ISFET using Si substrate.
\end{abstract}

Key words: semiconductor, ISFET, sensitivity, $\mathrm{pH}$, electrolyte

\section{Introduction}

Ion Sensitive Field Effect Transistor (ISFET) has been used for sensing chemical properties of solution. It has been used for monitoring the activity of ions in an electrolyte placed to the gate of a Metal-Oxide-Semiconductor structure. The concentration of hydrogen $(\mathrm{pH})$ in the chemicals is among the main parameters to be measured. The important advantages of using ISFET are lab-on-a-chip integration possibility and compatibility with the existing CMOS technologies [1]. Figure 1 illustrates the cross section of ISFET. This structure is similar to the structure of normal Metal-Oxide-Semiconductor Field-Effect Transistor (MOSFET), with the difference that instead of metal gate electrode, an electrolyte and an electrode have been used. The ISFET principle of operation is based on donation or acceptance proton of Hydroxyls at the interface of ion-solid between the sensitive layer and the electrolyte. [2].

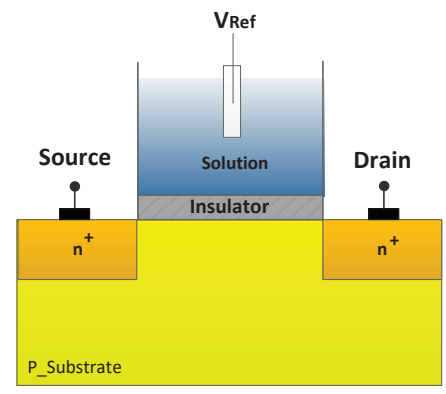

Fig.1.Structure of an ISFET
In this structure, by varying the $\mathrm{pH}$, load distribution in the gate insulator is changed and so the ID of the discharge current will change [2]. In this paper TCAD-based methodology used for the simulation of ISFET and sensor sensitivity enhancement by changing substrate material.

\section{Theory of ISFET and Simulation Method}

ISFET operation and mechanism responsible for the change in surface charge is explained using site-binding theory [3]. According to this theory, the insulating surface has hydroxyl groups $(\mathrm{OH})$ which forms binding sites which acquire $\mathrm{H}^{+}$(thus positively charged) or losing $\mathrm{H}^{+}$ (thus negatively charged). The distribution of ions in the electrolyte is explained using GouyChapman-Stern (GCS) theory [3]. According to this theory electrolyte ions form two layers, Helmholtz layer and diffusion layer (Figure 2). Helmholtz layer is consisted of two regions.

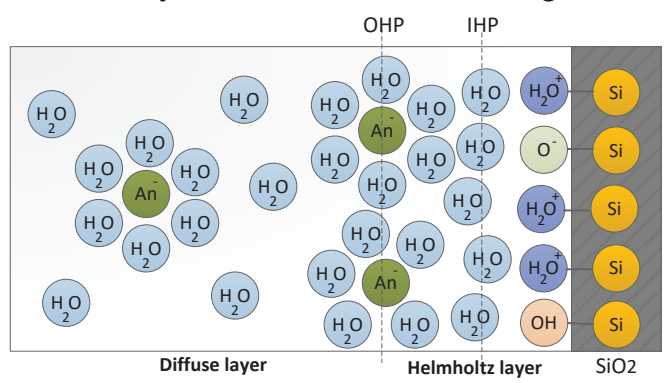

Fig.2. GCS model of the electrical double layer at the electrolyte-insulator interface. 
The first region is formed from insulatorelectrolyte surface to the Inner Helmholtz Plan (IHP), which is the locus of centers of the specifically adsorbed ions. Next region is formed from IHP to outer Helmholtz plane (OHP) which is the locus of the centers of the hydrated ions at their distance of closest approach to the solid. The diffuse layer is formed from OHP to the bulk of solution [3].

The charge distribution in ISFET consists of the charge in the semiconductor channel, surface charge on the Insulator-Electrolyte interface due to ion adsorbing and charge distribution through the diffuse layer. By solving the charge and potential equations of different regions, ID related of each $\mathrm{pH}$ is obtained [4].

Numerical simulation tools are used to solve ISFET charge and potential equations .In this case, the sensor sensitivity is given by [4]:

$$
S=\left|\frac{\partial I_{D}}{\partial p H}\right|_{V_{\operatorname{Re} f}(\text { CONSTANT })}
$$

\section{Result and Discussion}

The simulation of ISFET device is performed using the method described in last section to study the effect of properties of ISFET $\mathrm{pH}$ sensor on the output current and sensitivity. In order to check the simulation validity, the reference voltage is tuned to $1 \mathrm{~V}$ and the drain current $\mathrm{I}_{\mathrm{D}}$ is extracted versus $\mathrm{pH}$ curve and set the drain current $I_{D}$ to $10 \mu \mathrm{A}$ and extracted the reference voltage versus $\mathrm{pH}$ curve and compared the results with the data in [4] and shown in figure 3.
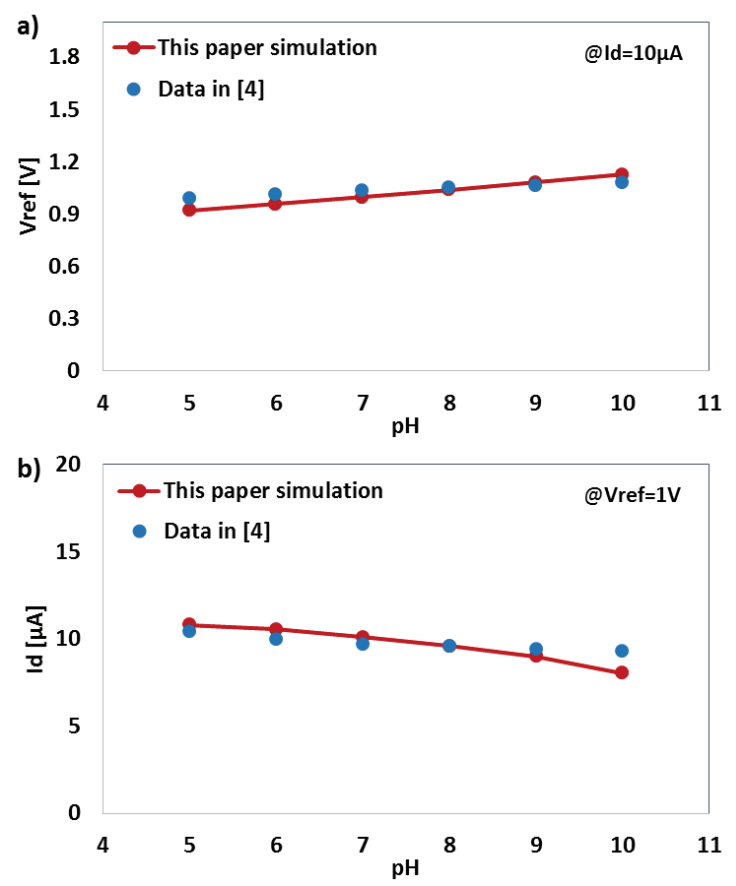

Fig.3. a) $V_{\text {Ref versus }} p H$ and b) $I_{D}$ versus $p H$ Compared with data in [4].
In order to investigate and improve ISFET sensitivity, the sensitivity results obtained from the equation (1) with $\mathrm{Si}$ and GaAs substrate are presented in figure 4 . It is observed that ISFET with GaAs substrate has in average 2.3 times more sensitivity against $\mathrm{pH}$ changes than ISFET with Si substrate.

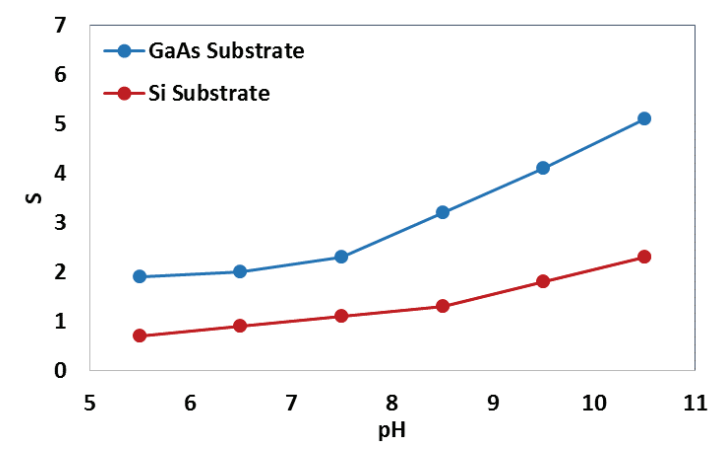

Fig.4. ISFET sensitivity, $S$ versus $p H$ value with Si and GaAs substrate.

\section{Conclusion}

In this paper, a methodology based on charge and potential equations of an electrolyteinsulator-semiconductor structure is introduced and simulated by device simulations. Sensitivity of this sensor was defined as the drain current change in per $\mathrm{pH}$ unit. Moreover, to gain high sensitivity, material of substrate was changed. Results showed that ISFET with GaAs substrate has in average 2.3 times more sensitivity against $\mathrm{pH}$ changes than ISFET with Si substrate.

\section{References}

[1] Choksi, Neel, Dewanshu Sewake, Soumendu Sinha, Ravindra Mukhiya, and Rishi Sharma. "Modeling and simulation of ion-sensitive fieldeffect transistor using TCAD methodology," in Electronics, Materials Engineering and NanoTechnology (IEMENTech),(2017):1-4; doi: 10.1109/IEMENTECH.2017.8076935

[2] Bandiziol, Andrea, Pierpaolo Palestri, Federico Pittino, David Esseni, and Luca Selmi. "A TCADbased methodology to model the site-binding charge at ISFET/electrolyte interfaces," IEEE Transactions on Electron Devices, 62(2015):3379-3386; doi: 10.1109/TED.2015.2464251

[3] Dutta, Jiten Ch, "Ion sensitive field effect transistor for applications in bioelectronic sensors: A research review," in Computational Intelligence and Signal Processing (CISP),(2012): 185-191; doi: 10.1109/NCCISP.2012.6189704

[4] Abdolkader, Tarek M., Abdurrahman G. Alahdal, Ahmed Shaker, and Wael Fikry. "ISFET pHsensor sensitivity extraction using conventional MOSFET simulation tools," International Journal of Chemical Engineering and Applications, 6(2015):346; doi: 10.7763/IJCEA.2015.V6.507 